Bull. Austral. Math. Soc.

VOL. 57 (1998) [173-174]

\title{
ON THE CRITICAL POINTS OF A POLYNOMIAL
}

\author{
ABDUl AzIZ AND B.A. ZaRgar
}

Let $p$ be a complex polynomial, of the form $p(z)=z \prod_{k=1}^{n-1}\left(z-z_{k}\right)$, where $\left|z_{k}\right| \geqslant 1$ when $1 \leqslant k \leqslant n-1$. Then $p^{\prime}(z) \neq 0$ if $|z|<1 / n$.

Let $B(z, r)$ denote the open ball in $\mathbb{C}$ with centre $z$ and radius $r$, and $\bar{B}(z, r)$ denote its closure. The Gauss-Lucas theorem states that every critical point of a complex polynomial $p$ of degree at least 2 lies in the convex hull of its zeros. This theorem has been further investigated and developed. B. Sendov conjectured that, if all the zeros of $p$ lie in $\bar{B}(0,1)$, then, for any zero $\zeta$ of $p$, the disc $\bar{B}(\zeta, 1)$ contains at least one zero of $p^{\prime}$; see [3, Problem 4.1]. This conjecture has attracted much attention-see, for example, [1], and the papers cited there. In connection with this conjecture, Brown [2] posed the following problem.

Let $\mathcal{Q}_{n}$ denote the set of all complex polynomials of the form $p(z)=z \prod_{k=1}^{n-1}\left(z-z_{k}\right)$, where $\left|z_{k}\right| \geqslant 1$ when $1 \leqslant k \leqslant n-1$. Find the best constant $C_{n}$ such that $p^{\prime}$ does not vanish in $B\left(0, C_{n}\right)$, for all $p$ in $\mathcal{Q}_{n}$.

Brown observed that, if $p(z)=z(z-1)^{n-1}$, then $p^{\prime}(1 / n)=0$, and conjectured that $C_{n}=1 / n$. We show this here.

THEOREM For all $p$ in $\mathcal{Q}_{n}, p^{\prime}(z) \neq 0$ if $z \in B(0,1 / n)$. and so

ProOF: Clearly $p^{\prime}(0)=\prod_{k=1}^{n-1}\left(-z_{k}\right) \neq 0$. If $0<|z|<1 / n$, then $\left|z-z_{k}\right|>1-1 / n$,

$$
\left|\frac{p^{\prime}(z)}{p(z)}\right|=\left|\frac{1}{z}+\sum_{k=1}^{n-1} \frac{1}{z-z_{k}}\right| \geqslant \frac{1}{|z|}-\sum_{k=1}^{n-1} \frac{1}{\left|z-z_{k}\right|}>n-\sum_{k=1}^{n-1} \frac{n}{n-1}=0 .
$$

It follows that $p^{\prime}$ does not vanish in $B(0,1 / n)$.

Similarly, if $p(z)=z^{m} \prod_{k=1}^{n-m}\left(z-z_{k}\right)$, where $\left|z_{k}\right| \geqslant 1$ when $1 \leqslant k \leqslant n-m$, then $p^{\prime}(z) \neq 0$ if $0<|z|<m / n$.

Received 1st September, 1997

A paper by these two authors was submitted to the Bulletin of the Australian Mathematical Society on November 4,1996 . The shortened proof here is due to the Editor. However the above-named authors should have the credit for this result.

Copyright Clearance Centre, Inc. Serial-fee code: 0004-9729/98 \$Ä2.00+0.00. 


\section{REFERENCES}

[1] Iulius Borcea, 'On the Sendov conjecture for polynomials with at most six distinct roots', J. Math. Anal. Appl. 200 (1996), 182-206.

[2] J.E. Brown, 'On the Ilief-Sendov conjecture', Pacific J. Math. 135 (1988), 223-232.

[3] W.K. Hayman, Research problems in function theory (Athlone Press, London, 1967).

Postgraduate Department of Mathematics

University of Kashmir

Hazratbal

Srinigar 190006

India 